\title{
Strategy-proof division with single-peaked preferences and initial endowments
}

Citation for published version (APA):

Klaus, B. E., Storcken, A. J. A., \& Peters, H. J. M. (1995). Strategy-proof division with single-peaked preferences and initial endowments. METEOR, Maastricht University School of Business and Economics. METEOR Research Memorandum No. 001 https://doi.org/10.26481/umamet.1995001

Document status and date:

Published: 01/01/1995

DOI:

10.26481/umamet.1995001

Document Version:

Publisher's PDF, also known as Version of record

\section{Please check the document version of this publication:}

- A submitted manuscript is the version of the article upon submission and before peer-review. There can be important differences between the submitted version and the official published version of record.

People interested in the research are advised to contact the author for the final version of the publication, or visit the DOI to the publisher's website.

- The final author version and the galley proof are versions of the publication after peer review.

- The final published version features the final layout of the paper including the volume, issue and page numbers.

Link to publication

\footnotetext{
General rights rights.

- You may freely distribute the URL identifying the publication in the public portal. please follow below link for the End User Agreement:

www.umlib.nl/taverne-license

Take down policy

If you believe that this document breaches copyright please contact us at:

repository@maastrichtuniversity.nl

providing details and we will investigate your claim.
}

Copyright and moral rights for the publications made accessible in the public portal are retained by the authors and/or other copyright owners and it is a condition of accessing publications that users recognise and abide by the legal requirements associated with these

- Users may download and print one copy of any publication from the public portal for the purpose of private study or research.

- You may not further distribute the material or use it for any profit-making activity or commercial gain

If the publication is distributed under the terms of Article $25 \mathrm{fa}$ of the Dutch Copyright Act, indicated by the "Taverne" license above, 


\title{
Strategy-proof division with single-peaked preferences and initial endowments
}

\author{
Bettina Klaus Hans Peters Ton Storcken*
}

January 1995

\begin{abstract}
We consider the problem of (re)allocating the total endowment of an infinitely divisible commodity among agents with single-peaked preferences and initial endowments. We propose an extension of the so-called uniform rule and show that it is the unique rule satisfying strategy-proofness, Pareto optimality, and an equal-treatment condition. This last condition is implied by the combination of anonymity and translation invariance, which fact is used to obtain a second characterization. The resulting rule turns out to be peaks-only and individually rational: the allocation assigned by the rule depends only on the peaks of the preferences, and no agent is worse off than at his initial endowment.

Journal of Economic Literature Classification Numbers: D63, D71.
\end{abstract}

\section{Introduction}

Consider the problem of allocating teaching hours among the members of a university department. It is reasonable to assume that preferences for teaching are single-peaked: each individual has an optimally preferred number of teaching hours, below which and above which preference is decreasing. The existing distribution of teaching hours may be unsatisfactory, for instance because preferences have changed over time. Then the question arises how to reallocate teaching hours.

The special instance of this problem in which only the total endowment plays a role (and, consequently, initial endowments are not modeled), has been studied extensively in economic literature. The allocation rule featuring preeminently in this literature is the so-called "uniform rule". This rule was already described as a strategy-proof rationing scheme by Benassy (1982). Sprumont (1991) showed that it is the unique Pareto optimal, anonymous, and strategyproof rule. As usual, strategy-proofness means that no agent can gain by misrepresenting his preferences. Anonymity implies that only the preferences and not the names of the agents matter. Ching (1994) weakens anonymity to a condition called equal treatment of equals. Other characterizations of the uniform

*Department of Quantitative Economics, University of Limburg, P.O. Box 616, 6200 MD Maastricht, The Netherlands 
rule were obtained by Thomson (1991, 1994a, 1994b), using monotonicity and consistency properties, Otten et al. (1995), applying conditions from bargaining theory, and Angeles de Frutos and Masso (1994), using a condition called Lorenz maximality.

In this paper we consider the more general setting where initial endowments do play a role (not just through the total endowment). There are several ways in which initial endowments may influence the allocation. An agent who, at the reallocation assigned by the rule to be used, is worse off than at his initial endowment, might refuse to participate in the reallocation operation if he has the chance; indeed, just applying the uniform rule in a problem with initial endowments may lead to non-individually rational allocations. Likewise, an agent's net demand, i.e., the difference between his reported preference peak and his initial endowment, may be considered relevant, rather than his mere preference or preference peak. We will formalize this latter consideration as an equal-treatment condition. Besides, we impose Pareto optimality and strategyproofness with respect to the reported preferences - the initial endowments are assumed to be publicly known-to obtain what will be called the "uniform reallocation rule". This rule turns out to be individually rational as well. Furthermore, we show that the equal-treatment condition may be replaced by anonymity and translation invariance.

Besides in situations as the one at the beginning of this introduction, the uniform reallocation rule can be used in exchange economies with two goods and fixed prices, where rationing of one of the goods entails an allocation of the other good, in view of fixed prices and budgets. For more than two goods, one needs a multi-dimensional rule.

The uniform reallocation rule can be seen as an extension of the uniform rule for problems without initial endowments, by starting from equal division of the amount to be divided in such problems. The proof of our main characterization is structured in a similar way as Ching's (1994) elegant proof for the uniform rule. We also show that Pareto optimality and strategy-proofness imply own-peak monotonicity and uncompromisingness. The first property means that increasing the peak of an agent while leaving the endowments unchanged, does not decrease the amount allocated to that agent. The second condition implies that the allocation of an agent does not change if the peak of that agent remains at the same side of the allocation. Ching's result and ours are, however, logically independent, and-except for the global structure - the proofs are rather different.

The organization of the paper is as follows. In Section 2 we formulate the model and the uniform reallocation rule with its main properties. In Section 3 we state and prove the main characterization of the uniform reallocation rule. Section 4 is devoted to a variation on this characterization, and to showing independence of the axiom systems. 


\section{The uniform reallocation rule}

Let $N=\{1,2, \ldots, n\}$ denote the set of agents. Each agent $i \in N$ has a singlepeaked preference on $I R$, i.e. a complete and transitive binary relation $R_{i}$ on $I R$ for which there exists a point $p_{i} \in \mathbb{I}_{+}$with $^{1}$ the following property: for all $\alpha, \beta \in \mathbb{R}$ with $\beta<\alpha \leq p_{i}$ or $\beta>\alpha \geq p_{i}$ we have $\alpha P_{i} \beta$, where $P_{i}$ is the asymmetric part of $R_{i}$. As usual, $\alpha R_{i} \beta$ is interpreted as " $\alpha$ is weakly preferred to $\beta$ ", and $\alpha P_{i} \beta$ as " $\alpha$ is strictly preferred to $\beta$ ". The symmetric part of $R_{i}$ is denoted by $I_{i}: \alpha I_{i} \beta$ means that individual $i$ is indifferent between $\alpha$ and $\beta$. The point $p_{i}$ is called the peak of $R_{i}$ and will also be denoted by $p\left(R_{i}\right)$. By $\mathcal{R}$ we denote the class of all single-peaked preferences. An element $R=\left(R_{1}, \ldots, R_{n}\right)$ of $\mathcal{R}^{N}$ is called a preference profile. Furthermore, each agent $i$ has an initial endowment $e_{i} \in \mathbb{R}_{+}$. A division problem (or briefly: problem) is a pair $(e, R)$ where $e=\left(e_{1}, \ldots, e_{n}\right)$ is a vector of initial endowments and $R \in \mathcal{R}^{N}$ is a preference profile.

For $i \in N$, a preference $R_{i} \in \mathcal{R}$ and a number $\tau \in \mathbb{R}$ such that $p\left(R_{i}\right)+\tau \geq 0$, we define the preference $R_{i}+\tau$ by: for all $\alpha, \beta \in \mathbb{R}$

$$
\alpha\left(R_{i}+\tau\right) \beta \text { if }(\alpha-\tau) R_{i}(\beta-\tau)
$$

For a problem $(e, R)$, a (feasible) allocation is a vector $x \in \mathbb{R}_{+}^{N}$ with $\sum_{i=1}^{n} x_{i}=\sum_{i=1}^{n} e_{i}$. A rule is a map assigning to every problem a feasible allocation.

Let $\varphi$ be a rule. We are interested in the following possible properties of $\varphi$.

Pareto optimality For every problem $(e, R)$ there is no (feasible) allocation $x$ with $x_{i} R_{i} \varphi_{i}(e, R)$ for all $i \in N$ and $x_{i} P_{i} \varphi_{i}(e, R)$ for at least one $i \in N$.

Strategy-proofness For all $j \in N$ and all problems $(e, R),\left(e^{\prime}, R^{\prime}\right)$ with $e=e^{\prime}$ and $R_{i}=R_{i}^{\prime}$ for all $i \neq j$, we have $\varphi_{j}(e, R) R_{j} \varphi_{j}\left(e^{\prime}, R^{\prime}\right)$.

Equal-treatment For all $i, j \in N$, every $\tau \in I R$, and every problem $(e, R)$ with $R_{j}=R_{i}+\tau$ and $e_{j}=e_{i}+\tau$ we have

$$
\varphi_{j}(e, R) I_{j} \varphi_{i}(e, R)+\tau \text {. }
$$

Pareto optimality needs no further explanation. Strategy-proofness ensures that, if preferences are private information, in the game where each agent reports his preference it is a (weakly) dominant strategy to reveal one's true preference. Equal-treatment requires that, if the initial endowments and preferences of two agents are equal up to a translation, then each agent should be indifferent between his own allocation and the translated allocation of the other agent $^{2}$.

\footnotetext{
${ }^{1}$ The choice of $\mathbb{I} R_{+}$is not essential; $I R$ or some interval could be chosen as well.

${ }^{2}$ The uniform reallocation rule defined below actually satisfies the stronger version where we would have equality instead of indifference.
} 
A well-known rule, satisfying strategy-proofness and Pareto optimality - see Sprumont (1991) or Ching (1994), is the uniform rule $U$ defined ${ }^{3}$ by

$$
U_{j}(e, R):= \begin{cases}\min \left\{p\left(R_{j}\right), \lambda\right\} & \text { if } \sum_{i=1}^{n} p\left(R_{i}\right)>\sum_{i=1}^{n} e_{i} \\ \max \left\{p\left(R_{j}\right), \lambda\right\} & \text { if } \sum_{i=1}^{n} p\left(R_{i}\right) \leq \sum_{i=1}^{n} \epsilon_{i}\end{cases}
$$

for every $j \in N$, where $\lambda$ solves $\sum_{i=1}^{n} U_{i}(e, R)=\sum_{i=1}^{n} e_{i}$. The uniform rule does not take the individual initial distribution of the total endowment into account; for instance, it does not satisfy equal-treatment. As an alternative, we propose the uniform reallocation rule $U^{r}$ defined by

$$
U_{j}^{r}(e, R):= \begin{cases}\min \left\{p\left(R_{j}\right), e_{j}+\lambda\right\} & \text { if } \sum_{i=1}^{n} p\left(R_{i}\right)>\sum_{i=1}^{n} e_{i} \\ \max \left\{p\left(R_{j}\right), e_{j}-\lambda\right\} & \text { if } \sum_{i=1}^{n} p\left(R_{i}\right) \leq \sum_{i=1}^{n} e_{i}\end{cases}
$$

for every $j \in N$, where $\lambda \geq 0$ and $\lambda$ solves $\sum_{i=1}^{n} U_{i}^{r}(e, R)=\sum_{i=1}^{n} e_{i}$. Observe that the uniform reallocation rule is well defined: it assigns a feasible allocation to every division problem. It can be seen as an extension of the uniform rule in a sense specified by the following lemma.

Lemma 1 For every problem $(e, R)$

$$
U(e, R)=U^{r}(\bar{e}, R)
$$

where $\bar{e}=\left(\frac{E}{n}, \ldots, \frac{E}{n}\right), E:=\sum_{i=1}^{n} e_{i}$

In words: applying the uniform rule gives the same result as applying the uniform reallocation rule from equal initial endowments.

Proof Let $(e, R)$ be a division problem. Assume that $\sum_{i=1}^{n} p\left(R_{i}\right)>$ $\sum_{i=1}^{n} e_{i}=: E$, the other case is similar. For agents $i \in N$ such that $p\left(R_{i}\right) \leq \frac{E}{n}$ we have $U_{i}^{r}(\bar{e}, R)=p\left(R_{i}\right)=U_{i}(e, R)$. If $i \in N$ with $p\left(R_{i}\right)>\frac{E}{n}$, then $U_{i}^{r}(\bar{e}, R)=$ $\min \left\{p\left(R_{i}\right), \frac{E}{n}+\lambda\right\}$ where $\lambda$ solves $\sum_{i=1}^{n} U_{i}^{r}(\bar{e}, R)=E$. Let $\lambda^{\prime}:=\frac{E}{n}+\lambda$. Then, $U_{i}^{r}(\bar{e}, R)=\min \left\{p\left(R_{i}\right), \lambda^{\prime}\right\}$ where $\lambda^{\prime}$ solves $\sum_{i=1}^{n} U_{i}^{r}(\bar{e}, R)=E=\sum_{i=1}^{n} U_{i}(e, R)$. Hence, $U_{i}^{r}(\bar{e}, R)=U_{i}(e, R)$.

Let $\varphi$ be a rule with, for every problem $(e, R)$, either $\varphi_{i}(e, R) \leq p\left(R_{i}\right)$ for all $i \in N$ or $\varphi_{i}(e, R) \geq p\left(R_{i}\right)$ for all $i \in N$. We call such a rule same-sided. By single-peakedness of the preferences, it is easy to show that a same-sided rule is Pareto optimal, and that, conversely, every Pareto optimal rule must be same-sided. (Sprumont (1991) actually uses same-sidedness as definition of Pareto optimality.) For later reference, we state this observation as a lemma.

Lemma 2 A rule $\varphi$ is Pareto optimal if and only if it is same-sided.

\footnotetext{
${ }^{3}$ This definition is adapted to our context. In the original literature only the total endowment is specified and no initial endowments.
} 


\section{Main characterization}

This section is entirely devoted to the following characterization of the uniform reallocation rule.

Theorem 3 The uniform reallocation rule is the unique rule satisfying Pareto optimality, strategy-proofness, and equal-treatment.

In what follows it is useful to distinguish between suppliers and demanders in a division problem $(e, R)$. The set of suppliers is defined as $S(e, R):=\{i \in$ $\left.N \mid p\left(R_{i}\right) \leq e_{i}\right\}$ and the set of demanders as $D(e, R):=\left\{i \in N \mid p\left(R_{i}\right)>e_{i}\right\}$. If it is clear which division problem is meant, also the notations $S(p)$ and $D(p)$ will be used, where $p$ is the vector of peaks ${ }^{4}$.

We start by showing that the uniform reallocation rule has all the properties mentioned in the theorem.

Proposition 4 The uniform reallocation rule is Pareto optimal, strategy-proof, and satisfies equal-treatment.

Proof. Pareto optimality of $U^{r}$ follows immediately from same-sidedness, see Lemma 2.

In order to show strategy-proofness of $U^{r}$, let $j \in N$ and let $(e, R)$ and $\left(e, R^{\prime}\right)$ be division problems with $R_{i}=R_{i}^{\prime}$ for all $i \neq j$. We have to prove that

$$
U_{j}^{r}(e, R) R_{j} U_{j}^{r}\left(e, R^{\prime}\right)
$$

For all agents $i$ write $p_{i}:=p\left(R_{i}\right), p_{i}^{\prime}:=p\left(R_{i}^{\prime}\right), E:=\sum_{i=1}^{n} e_{i}$. We assume that $\sum_{i=1}^{n} p_{i}>E$, the other case is similar. Then, $U_{j}^{r}(e, R)=\min \left\{p_{j}, e_{j}+\lambda\right\}$ where $\lambda \geq 0$ solves $\sum_{i=1}^{n} U_{i}^{r}(e, R)=E$. If $U_{j}^{r}(e, R)=p_{j}$, then (1) holds because $p_{j}$ is the peak of $R_{j}$; so we are done. Otherwise, $U_{j}^{r}(e, R)=e_{j}+\lambda$, i.e., agent $j$ is a demander. We distinguish two cases.

Case $1 p_{j}^{\prime}>e_{j}+\lambda$

Observe that in the profile $R^{\prime}$ agent $j$ is still a demander. Consequently, $D\left(e, R^{\prime}\right)=D(e, R)$ and by feasibility and same-sidedness

$$
\begin{aligned}
\sum_{i \in D\left(e, R^{\prime}\right)} p_{i}^{\prime}-e_{i} & =p_{j}^{\prime}-e_{j}+\sum_{i \in D(e, R), i \neq j} p_{i}-e_{i} \\
& >\lambda+\sum_{i \in D(e, R), i \neq j} U_{i}^{r}(e, R)-e_{i} \\
& =\sum_{i \in D(e, R)} U_{i}^{r}(e, R)-e_{i} \\
& =\sum_{i \in S(e, R)} e_{i}-p_{i} \\
& =\sum_{i \in S\left(e, R^{\prime}\right)} e_{i}-p_{i}^{\prime} .
\end{aligned}
$$

\footnotetext{
${ }^{4}$ The inclusion of agents with peaks equal to their initial endowments among the suppliers is arbitrary, but convenient for what follows.
} 
Hence, $\sum_{i=1}^{n} p_{i}^{\prime}>E$. Therefore, $U_{j}^{r}\left(e, R^{\prime}\right)=\min \left\{p_{j}^{\prime}, e_{j}+\lambda^{\prime}\right\}$ where $\lambda^{\prime} \geq 0$ solves $\sum_{i=1}^{n} U_{i}^{r}\left(e, R^{\prime}\right)=E$. Because $p_{j}^{\prime}>e_{j}+\lambda$ and $p_{i}^{\prime}=p_{i}$ for $i \neq j$ it follows that $\lambda^{\prime}=\lambda$. Hence, $U_{j}^{r}\left(e, R^{\prime}\right)=e_{j}+\lambda=U_{j}^{r}(e, R)$, and (1) follows.

Case $\mathscr{2} p_{j}^{\prime} \leq e_{j}+\lambda$

If $\sum_{i=1}^{n} p_{i}^{\prime}>E$, then $U_{j}^{r}\left(e, R^{\prime}\right)=\min \left\{p_{j}^{\prime}, e_{j}+\lambda^{\prime}\right\} \leq e_{j}+\lambda=U_{j}^{r}(e, R) \leq p_{j}$. If $\sum_{i=1}^{n} p_{i}^{\prime} \leq E$, then $U_{j}^{r}\left(e, R^{\prime}\right)=\max \left\{p_{j}^{\prime}, e_{j}-\lambda^{\prime}\right\} \leq e_{j}+\lambda=U_{j}^{r}(e, R) \leq p_{j}$. So in both cases (1) holds.

The proof of equal-treatment of $U^{r}$ is straightforward and left to the reader.

In order to prove the converse direction of Theorem 3 we introduce the following two possible properties of a rule $\varphi$.

Own-peak monotonicity For every $j \in N$ and all problems $(e, R)$ and $\left(\epsilon^{\prime}, R^{\prime}\right)$ with $e=\epsilon^{\prime}, R_{i}=R_{i}^{\prime}$ for all $i \neq j$ and $p\left(R_{j}^{\prime}\right) \leq p\left(R_{j}\right)$ :

$$
\varphi_{j}\left(e^{\prime}, R^{\prime}\right) \leq \varphi_{j}(e, R)
$$

Uncompromisingness For every $j \in N$ and all problems $(e, R)$ and $\left(e^{\prime}, R^{\prime}\right)$ with $e=e^{\prime}, R_{i}=R_{i}^{\prime}$ for all $i \neq j$ : if $p\left(R_{j}\right)<\varphi_{j}(e, R)$ and $p\left(R_{j}^{\prime}\right) \leq \varphi_{j}(e, R)$ or if $p\left(R_{j}\right)>\varphi_{j}(e, R)$ and $p\left(R_{j}^{\prime}\right) \geq \varphi_{j}(e, R)$ then $\varphi_{j}(e, R)=\varphi_{j}\left(e^{\prime}, R^{\prime}\right)$.

Own-peak monotonicity says that an agent's allocation can never move in the opposite direction of a movement of his peak. Uncompromisingness states that, if an agent's peak differs from his allocation assigned by the rule and is moved without crossing that allocation, then that allocation does not change. Ownpeak monotonicity was introduced by Ching (1994). Uncompromisingness is a well-known property in connection with strategy-proofness (see for instance Border and Jordan (1983) in the context of public goods). Both properties are convenient to work with because they only use information concerning the peaks of the preferences. In the following two lemmas it is shown that both properties are implied by Pareto optimality and strategy-proofness.

Lemma 5 Let $\varphi$ be a Pareto optimal and strategy-proof rule. Then $\varphi$ is ownpeak monotonic.

Proof. Let $j \in N$ and let $(e, R)$ and $\left(e, R^{\prime}\right)$ be division problems with $R_{i}=R_{i}^{\prime}$ for all $i \neq j$, and $p\left(R_{j}^{\prime}\right) \leq p\left(R_{j}\right)$. We wish to show that $\varphi_{j}\left(e, R^{\prime}\right) \leq \varphi_{j}(e, R)$. For all agents $i$ write $p_{i}:=p\left(R_{i}\right), p_{i}^{\prime}:=p\left(R_{i}^{\prime}\right), E:=\sum_{i=1}^{n} e_{i}$. We distinguish two cases.

Case $1 \quad \sum_{i=1}^{n} p_{i}>E$

If $\sum_{i=1}^{n} p_{i}^{\prime} \geq E$, then feasibility and same-sidedness (see Lemma 2) imply $\varphi_{i}\left(e, R^{\prime}\right) \leq p_{i}^{\prime}$ and $\varphi_{i}(e, R) \leq p_{i}$ for all $i \in N$. Hence $\varphi_{j}\left(e, R^{\prime}\right) \leq p_{j}$ and $\varphi_{j}(e, R) \leq p_{j}$. Because $\varphi_{j}(e, R) R_{j} \varphi_{j}\left(e, R^{\prime}\right)$ by strategy-proofness, it follows that $\varphi_{j}\left(e, R^{\prime}\right) \leq \varphi_{j}(e, R)$. 
If $\sum_{i=1}^{n} p_{i}^{\prime}<E$, then by same-sidedness and feasibility $p_{i}^{\prime} \leq \varphi_{i}\left(e, R^{\prime}\right)$ and $p_{i} \geq \varphi_{i}(e, R)$ for all $i \in N$. Therefore $p_{j}^{\prime} \leq \varphi_{j}\left(e, R^{\prime}\right)$. Now

$$
p_{j}^{\prime}<E-\sum_{i=1, i \neq j}^{n} p_{i}=\sum_{i=1, i \neq j}^{n}\left(\varphi_{i}(e, R)-p_{i}\right)+\varphi_{j}(e, R) \leq \varphi_{j}(e, R) .
$$

Because $\varphi_{j}\left(e, R^{\prime}\right) R_{j}^{\prime} \varphi_{j}(e, R)$ by strategy-proofness, it follows that $\varphi_{j}\left(e, R^{\prime}\right) \leq$ $\varphi_{j}(e, R)$.

Case $2 \sum_{i=1}^{n} p_{i} \leq E$

In this case we have $\sum_{i=1}^{n} p_{i}^{\prime} \leq E$. By feasibility and same-sidedness we have $p_{j}^{\prime} \leq \varphi_{j}\left(e, R^{\prime}\right)$ and $p_{j} \leq \varphi_{j}(e, R)$. So $p_{j}^{\prime} \leq \varphi_{j}(e, R)$.

Because $\varphi_{j}\left(e, R^{\prime}\right) R_{j}^{\prime} \varphi_{j}(e, R)$ by strategy-proofness, it follows that $\varphi_{j}\left(e, R^{\prime}\right) \leq$ $\varphi_{j}(e, R)$.

An immediate but important consequence of own-peak monotonicity of a rule $\varphi$ is individual peak-onliness, i.e., for every $j \in N, \varphi_{j}(e, R)=\varphi_{j}\left(e^{\prime}, R^{\prime}\right)$ whenever $e=e^{\prime}, R_{i}=R_{i}^{\prime}$ for all $i \neq j$, and $p\left(R_{j}\right)=p\left(R_{j}^{\prime}\right)$.

We proceed with the result concerning uncompromisingness.

Lemma 6 Let $\varphi$ be a Pareto optimal and strategy-proof rule. Then $\varphi$ is uncompromising.

Proof Let $j \in N$ and let $(e, R),\left(e, R^{\prime}\right)$ be division problems with $R_{i}=R_{i}^{\prime}$ for all $i \neq j, p\left(R_{j}\right)>\varphi_{j}(e, R)$, and $p\left(R_{j}^{\prime}\right) \geq \varphi_{j}(e, R)$ (the other case is similar). We wish to prove that $\varphi_{j}(e, R)=\varphi_{j}\left(e, R^{\prime}\right)$. For all agents $i$ write $p_{i}:=p\left(R_{i}\right)$, $p_{i}^{\prime}:=p\left(R_{i}^{\prime}\right), E:=\sum_{i=1}^{n} e_{i}$.

If $\varphi_{j}\left(e, R^{\prime}\right)<\varphi_{j}(e, R)$ then $\varphi_{j}(e, R) P_{j}^{\prime} \varphi_{j}\left(e, R^{\prime}\right)$, violating strategy-proofness. Therefore, $\varphi_{j}\left(e, R^{\prime}\right) \geq \varphi_{j}(e, R)$.

Assume that $R_{j}$ is a preference which is symmetric around its peak: because $\varphi$ is own-peak monotonic by Lemma 5 and therefore individually peak-only, this is without loss of generality. If $\varphi_{j}\left(e, R^{\prime}\right)>\varphi_{j}(e, R)$ then $\varphi_{j}\left(e, R^{\prime}\right) \geq 2 p_{j}-$ $\varphi_{j}(e, R)$ because otherwise $\varphi_{j}\left(e, R^{\prime}\right) P_{j} \varphi_{j}(e, R)$, violating strategy-proofness. By Pareto optimality this implies $p_{j}^{\prime} \geq \varphi_{j}\left(e, R^{\prime}\right) \geq 2 p_{j}-\varphi_{j}(e, R)$. Hence, as long as $p_{j}^{\prime}<2 p_{j}-\varphi_{j}(e, R)$ we have $\varphi_{j}\left(e, R^{\prime}\right)=\varphi_{j}(e, R)$. By repeating this argument, each time shifting agent's $j$ 's peak to the right, we obtain $\varphi_{j}\left(e, R^{\prime}\right)=\varphi_{j}(e, R)$ for any location of $p_{j}^{\prime}$.

Remark 1 The repetition argument in the proof of Lemma 6 can be avoided by taking a sufficiently asymmetric preference $R_{j}$ instead (cf. Ching, 1994, Lemma 2). We deliberately used the above argument to be able to conclude later (see Remark 3 ) that Theorem 3 remains valid if only symmetric preferences are allowed.

Our next task is to prove the converse of Proposition 4.

Proposition 7 Let $\varphi$ be a rule satisfying Pareto optimality, strategy-proofness, and equal-treatment. Then $\varphi$ is the uniform reallocation rule $U^{r}$. 
Proof We assume that $\varphi \neq U^{r}$ and derive a contradiction. Let $(e, R)$ be a division problem with $\varphi(e, R) \neq U^{r}(e, R)$. Assume that $\sum_{i=1}^{n} p\left(R_{i}\right)>E:=$ $\sum_{i=1}^{n} e_{i}$ (the other case can be handled in a similar way).

By Lemmas 5 and 6 both $\varphi$ and $U^{r}$ are own-peak monotonic and uncompromising.

Let $m \in \arg \max \left\{p\left(R_{i}\right)-e_{i} \mid i \in D(e, R)\right\}$, and let $M(e, R):=\left\{i \in D(e, R) \mid R_{i}=\right.$ $\left.R_{m}+\tau_{i},\right\}$, where $\tau_{i}:=e_{i}-e_{m}$. In words, agent $m$ is an arbitrary but fixed agent with maximal demand, and $M(e, R)$ is the set of maximal demanders that have the same preferences as agent $m$ up to a translation; so $M(e, R)$ contains at least $m$.

We say that $\Gamma(e, R)$ holds if the following two conditions are satisfied:

(i) For all $i \in S(e, R): \varphi_{i}(e, R)=p\left(R_{i}\right)$.

(ii) $D(e, R)=M(e, R)$.

Suppose $\Gamma(e, R)$ holds. Then, by definition, for all $i \in S(e, R): U_{i}^{r}(e, R)=$ $p\left(R_{i}\right)$. With (i) and feasibility this implies

$$
\sum_{i \in D(e, R)} U_{i}^{r}(e, R)=\sum_{i \in D(e, R)} \varphi_{i}(e, R) .
$$

By (ii) and equal-treatment therefore $U_{i}^{r}(e, R)=\varphi_{i}(e, R)$ for all $i \in D(e, R)$. Hence, $U^{r}(e, R)=\varphi(e, R)$, violating the assumption $\varphi(e, R) \neq U^{r}(e, R)$. This contradiction completes the proof for the case that $\Gamma(e, R)$ holds. Otherwise, we have the following claim:

Claim If $\Gamma(e, R)$ does not hold, then there is a problem $\left(e, R^{\prime}\right)$ satisfying the following two conditions:

(iii) $M\left(e, R^{\prime}\right) \supset M(e, R), M\left(e, R^{\prime}\right) \neq M(e, R)$.

(iv) $\varphi\left(e, R^{\prime}\right) \neq U^{r}\left(e, R^{\prime}\right)$.

We will prove this claim below. First observe that for $\left(e, R^{\prime}\right)$ as in the claim $\Gamma\left(e, R^{\prime}\right)$ cannot be true, because otherwise (iv) would be violated. Hence, by repeated application of the claim we can find an infinite sequence of division problems satisfying (iii) and (iv) but not both (i) and (ii). By (iii), however, the number of maximal demanders with the same preferences as $m$ up to a translation increases at every step, an obvious impossibility since $N$ is finite. So we have a contradiction.

We are left to prove the Claim. Suppose that $\Gamma(e, R)$ does not hold. We distinguish two cases.

Case 1 There exists a $k \in S(e, R)$ with $\varphi_{k}(e, R) \neq p\left(R_{k}\right)$. Then, by samesidedness and feasibility, $\varphi_{k}(e, R)<p\left(R_{k}\right)$. Define $R_{i}^{\prime}:=R_{i}$ for all $i \neq k$ and $R_{k}^{\prime}:=R_{m}+\tau_{k}$. In other words, we turn agent $k$ into an agent in $M\left(e, R^{\prime}\right)$, so that the number of maximal demanders that up to a translation have the same preferences as $m$, is increased. By uncompromisingness, $\varphi_{k}\left(e, R^{\prime}\right)=\varphi_{k}(e, R)$, hence $\varphi_{k}\left(e, R^{\prime}\right)<e_{k} \leq U_{k}^{r}\left(e, R^{\prime}\right)$. So also $\varphi\left(e, R^{\prime}\right) \neq U^{r}\left(e, R^{\prime}\right)$. 
Case 2 For all $k \in S(e, R): \varphi_{k}(e, R)=p\left(R_{k}\right)$. Because $\Gamma(e, R)$ does not hold, we have $M(e, R) \neq D(e, R)$. Note that $A:=\left\{i \in D(e, R) \mid \varphi_{i}(e, R)>\right.$ $\left.U_{i}^{r}(e, R)\right\} \neq \emptyset$. We distinguish two subcases.

In the first subcase, there is a $j \in A$ with $j \notin k M(e, R)$, i.e., $R_{j} \neq R_{m}+\tau_{j}$. Define $R^{\prime}$ by $R_{i}^{\prime}:=R_{i}$ for all $i \neq j$ and $R_{j}^{\prime}:=R_{m}+\tau_{j}$. Then $p\left(R_{j}^{\prime}\right) \geq$ $p\left(R_{j}\right)$, so that by own-peak monotonicity $\varphi_{j}\left(e, R^{\prime}\right) \geq \varphi_{j}(e, R)>U_{j}^{r}(e, R)$. By uncompromisingness, $U_{j}^{r}\left(e, R^{\prime}\right)=U_{j}^{r}(e, R)$. Hence, $\varphi_{j}\left(e, R^{\prime}\right)>U_{j}^{r}\left(e, R^{\prime}\right)$, and in particular, $\varphi\left(e, R^{\prime}\right) \neq U^{r}\left(e, R^{\prime}\right)$.

In the second subcase, $A \subset M(e, R)$, i.e., there is no $j \in A$ with $R_{j} \neq$ $R_{m}+\tau_{j}$. By feasibility:

$$
\sum_{i \in D(e, R)} U_{i}^{r}(e, R)=E-\sum_{i \in S(e, R)} p\left(R_{i}\right)=\sum_{i \in D(e, R)} \varphi_{i}(e, R) .
$$

Hence, there exists a $j \in D(e, R) \backslash M(e, R)$ such that $p\left(R_{j}\right) \geq U_{j}^{r}(e, R)>$ $\varphi_{j}(e, R)$. Again, define $R^{\prime}$ by $R_{i}^{\prime}:=R_{i}$ for all $i \neq j$ and $R_{j}^{\prime}:=R_{m}+\tau_{j}$. Uncompromisingness of $\varphi$ implies $\varphi_{j}\left(e, R^{\prime}\right)=\varphi_{j}(e, R)$. Own-peak monotonicity of $U^{r}$ implies $U_{j}^{r}(e, R) \leq U_{j}^{r}\left(e, R^{\prime}\right)$. Hence, $U_{j}^{r}\left(e, R^{\prime}\right)>\varphi_{j}\left(e, R^{\prime}\right)$, and in particular, $\varphi\left(e, R^{\prime}\right) \neq U^{r}\left(e, R^{\prime}\right)$.

In both subcases, $\left(e, R^{\prime}\right)$ satisfies conditions (iii) and (iv). This completes the proof of the claim, and therefore of the proposition.

Proof of Theorem 3 Theorem 3 follows from Propositions 4 and 7 .

Remark 2 Theorem 3 shows that Pareto optimality, strategy-proofness, and equal-treatment together imply peak-onliness, i.e., $\varphi(e, R)=\varphi\left(e^{\prime}, R^{\prime}\right)$ whenever $e=e^{\prime}$ and $p\left(R_{i}\right)=p\left(R_{i}^{\prime}\right)$ for all $i \in N$.

\section{Another characterization, and independence of the axioms}

In this section we first present an alternative characterization of the uniform reallocation rule $U^{r}$ : equal-treatment can be replaced by anonymity and translation invariance. Second, we show logical independence of the axioms in both characterizations. Third, we will make an observation concerning the domain of preferences.

For a permutation $\sigma$ of $N$, a vector $x \in \mathbb{R}_{+}^{N}$ and a profile $R \in \mathcal{R}^{N}$, let $\sigma(x):=\left(x_{\sigma(1)}, \ldots, x_{\sigma(n)}\right)$ and $\sigma(R):=\left(R_{\sigma(1)}, \ldots, R_{\sigma(n)}\right)$. The following property of a rule $\varphi$ implies that an assigned allocation does not depend on the names of the agents.

Anonymity $^{5}$ For every permutation $\sigma$ of $N$ and every division problem $(e, R)$

$$
\varphi_{\sigma(i)}(e, R)=\varphi_{i}(\sigma(e), \sigma(R)) \text { for all } i \in N .
$$

\footnotetext{
${ }^{5}$ This condition is different from the anonymity condition in Sprumont (1991), which refers only to the preferences.
} 
For a preference profile $R \in \mathcal{R}^{N}$ and a vector $t \in \mathbb{R}^{N}$ such that $p\left(R_{i}\right)+t_{i} \geq 0$ for all $i$, we define the preference profile $R+t$ by: for all $i \in N,(R+t)_{i}:=R_{i}+t_{i}$.

Translation invariance For every division problem $(e, R)$ and every $t \in \mathbb{R}^{N}$ with $e+t, p(R)+t, \varphi(e, R)+t \in \mathbb{R}_{+}^{N}$ :

$$
\varphi(e+t, R+t)=\varphi(e, R)+t .
$$

Observe that a notion of translation invariance is already present in the equaltreatment condition. Moreover we have the following lemma.

Lemma 8 Let the mule $\varphi$ be anonymous and translation invariant. Then $\varphi$ satisfies equal-treatment.

Proof. Let $i, j \in N, \alpha \in I R$, and let problem $(e, R)$ satisfy $R_{j}=R_{i}+\alpha$ and $e_{j}=e_{i}+\alpha$. We have to prove

$$
\varphi_{j}(e, R) I_{j} \varphi_{i}(e, R)+\alpha .
$$

Define the translation vector $t$ by $t_{i}:=\alpha$ and $t_{k}=0$ for $k \neq i$. Let $e^{\prime}:=e+t$ and $R^{\prime}:=R+t$. By translation invariance

$$
\varphi_{i}\left(e^{\prime}, R^{\prime}\right)=\varphi_{i}(e, R)+t_{i}=\varphi_{i}(e, R)+\alpha
$$

and

$$
\varphi_{j}\left(e^{\prime}, R^{\prime}\right)=\varphi_{j}(e, R)+t_{j}=\varphi_{j}(e, R)
$$

Let $\sigma$ be a permutation of $N$ with $\sigma(i)=j, \sigma(j)=i$ and $\sigma(k)=k$ for $k \neq i, j$. By anonymity

$$
\varphi_{\sigma(i)}\left(e^{\prime}, R^{\prime}\right)=\varphi_{i}\left(\sigma\left(e^{\prime}\right), \sigma\left(R^{\prime}\right)\right)=\varphi_{j}\left(e^{\prime}, R^{\prime}\right) .
$$

Hence, $\varphi_{j}(e, R)=\varphi_{i}(e, R)+\alpha$, in particular $\varphi_{j}(e, R) I_{j} \varphi_{i}(e, R)+\alpha$.

With anonymity and translation invariance we obtain an alternative characterization of the uniform reallocation rule in the following theorem.

Theorem 9 The uniform reallocation rule is the unique rule satisfying strategy-proofness, Pareto optimality, anonymity, and translation invariance.

Proof. We leave verification of anonymity and translation invariance of $U^{r}$ to the reader; for the other properties, see Theorem 3.

The converse direction follows from Theorem 3 and Lemma 8.

Next, we discuss logical independence of the axiom systems in Theorems 3 and 9 . In this discussion we include consideration of the following property of a rule $\varphi$.

Individual rationality For every problem $(e, R)$ and every $i \in N$

$$
\varphi_{i}(e, R) R_{i} e_{i}
$$


In words, no individual should be worse off than at his initial endowment. All examples below will be individually rational; this indicates that, in general, individual rationality cannot replace any of the axioms used in Theorems 3 and 9.

Example 1 The following rule $\varphi$ is Pareto optimal and strategy-proof, but does not satisfy equal-treatment. It is anonymous, but not translation invariant. It is also individually rational. It is defined as follows. For a division problem $(e, R)$ with vector of peaks $p$ :

- if $\sum_{i=1}^{n} p_{i}>\sum_{i=1}^{n} e_{i}$ then $\varphi_{i}(e, R):=p_{i}$ if $i \in S(e, R)$; allocate $S:=$ $\sum_{i \in S(e, R)} e_{i}-p_{i}$ uniformly among the agent(s) in $D(e, R)$ with lowest initial endowment; if there is something left, allocate this uniformly among the agent(s) in $D(e, R)$ with second lowest endowment, etc.

- if $\sum_{i=1}^{n} p_{i} \leq \sum_{i=1}^{n} e_{i}$ then apply the uniform reallocation rule.

Example 2 The following rule $\varphi$ is Pareto optimal and satisfies equal-treatment, but is not strategy-proof. It is anonymous and translation invariant. It is also individually rational. It is defined as follows. For a division problem $(e, R)$ with vector of peaks $p$ :

- if $\sum_{i=1}^{n} p_{i}>\sum_{i=1}^{n} e_{i}$ then $\varphi_{i}(e, R):=p_{i}$ if $i \in S(e, R)$; allocate $S:=$ $\sum_{i \in S(e, R)} e_{i}-p_{i}$ among the agents in $D(e, R)$ by giving each agent $i \in$ $D(e, R)$ the amount $\tau e_{i}+(1-\tau) p_{i}$, where $0 \leq \tau \leq 1$ is determined by feasibility.

- if $\sum_{i=1}^{n} p_{i} \leq \sum_{i=1}^{n} e_{i}$ then apply the uniform reallocation rule.

Example 3 The following rule $\varphi$ satisfies equal-treatment and strategy-proofness but not Pareto optimality. It is anonymous, translation invariant, and individually rational. It is defined as follows. For every division problem $(e, R)$

$$
\varphi(e, R)=e
$$

Example 4 The following rule $\varphi$ satisfies Pareto optimality, strategy-proofness, translation invariance, but not anonymity (nor equal-treatment). It is also individually rational. It is defined as follows. For a division problem $(e, R)$ with vector of peaks $p$ :

- if $\sum_{i=1}^{n} p_{i}>\sum_{i=1}^{n} e_{i}$ then $\varphi_{i}(e, R):=p_{i}$ if $i \in S(e, R)$; allocate $S:=$ $\sum_{i \in S(e, R)} e_{i}-p_{i}$ among the agents in $D(e, R)$ as follows. If $1 \in D(e, R)$ then $\varphi_{1}(e, R):=\min \left\{p_{1}, e_{1}+S\right\}$ and distribute what remains of $\mathrm{S}$ uniformly among the other agents in $D(e, R)$. Otherwise, apply the uniform reallocation rule.

- if $\sum_{i=1}^{n} p_{i} \leq \sum_{i=1}^{n} e_{i}$ then apply the uniform reallocation rule.

We conclude with a remark concerning the domain of preferences. 
Remark 3 By going over the proofs - see also Remark 1-the reader may verify that our results, in particular Theorems 3 and 9 , remain valid if the domain of single-peaked preferences is replaced by the much smaller domain of all singlepeaked preferences that are symmetric around their peaks. Such preferences are completely described by their peaks. Consequently, on this domain a rule is peak-only by definition.

\section{References}

Angeles de Frutos, M., and J. Massó (1994): "More on the Uniform Rule: Equality and Consistency," Working Paper, Universitat Autònoma de Barcelona.

Benassy, J.P. (1982): The Economics of Market Disequilibrium. San Diego: Academic Press.

Border, K.C., and J.S. Jordan (1983): "Straightforward Elections, Unanimity and Phantom Voters," Review of Economic Studies, 50, 153-170.

Ching, S. (1994): "An Alternative Characterization of the Uniform Rule," Social Choice and Welfare, 11, 131-136.

Otten, G.-J., H. Peters, and O. Volij (1995): "Two Characterizations of the Uniform Rule for Division Problems with Single-Peaked Preferences," Economic Theory, forthcoming.

Sprumont, Y. (1991): "The Division Problem with Single-Peaked Preferences: A Characterization of the Uniform Allocation Rule," Econometrica, 59, 509519.

Thomson, W. (1991): "Population-Monotonic Solutions to the Problem of Fair Division when Preferences are Single-Peaked," Economic Theory, forthcoming.

Thomson, W. (1994): "Consistent Solutions to the Problem of Fair Division when Preferences are Single-Peaked," Journal of Economic Theory, 2, 219-245.

Thomson, W. (1994): "Resource-Monotonic Solutions to the Problem of Fair Division when Preferences are Single-Peaked," Social Choice and Welfare, 11, 205-223. 